\title{
COUPLING METHODS FOR SUPERCONDUCTING CH-CAVITIES *
}

\author{
H. Liebermann, H. Podlech, U. Ratzinger, A. Sauer, \\ Institut für Angewandte Physik, Frankfurt, Germany
}

\section{Abstract}

The cross-bar H-type $(\mathrm{CH})$ cavity is a multi-gap drift tube structure based on the H-210 mode currently under development at IAP Frankfurt [1]. Based on detailed numerical simulations a 19 cell prototype cavity from massive Niobium was realized. Experimental investigations have started already. With respect to rf coupling inductive coupling was applied successfully for cavity rf conditioning at room temperature with power levels up to 2 $\mathrm{kW}$. Capacitive coupling through the girder is however preferred in case of sc operation. Recent investigations led to a modified cavity geometry which allows to integrate a capacitive power coupler with $Q_{e}$ values down to $10^{6}$ in future $\mathrm{CH}$-cavities. Main consequences on the cavity are significantly lower and broader girder profiles as well as simplified stem geometries (round instead of elliptical stem bases). These modifications will provide space for power couplers through the girders. Additionally, the cavity stiffness against vacuum forces is improved by these modifications.

\section{SUPERCONDUCTING (SC) CH-PROTOTYPE}

In sc cavities there is no cooling problem as in cw operated $\mathrm{rt}$ (room temperatur) linacs. In general, sc linacs can be operated at higher gradients above a certain duty factor.

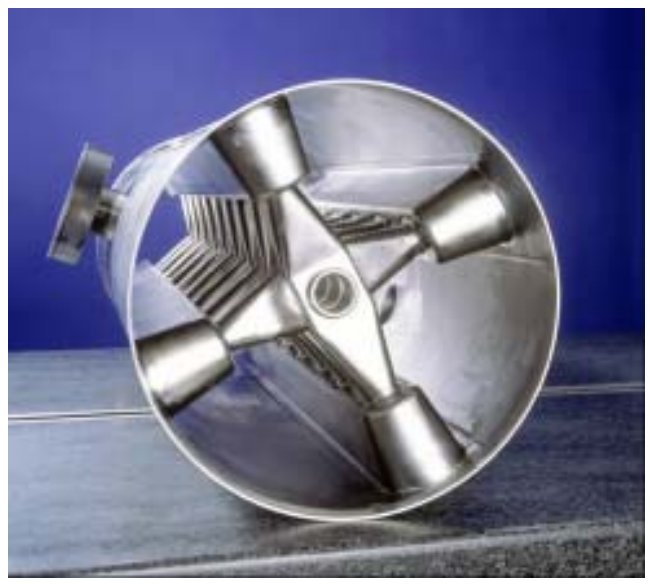

Figure 1: The sc $352 \mathrm{MHZ} \mathrm{CH}$ prototype

On the other hand, at low duty factors and high beam currents $\mathrm{rt}$ structures are very favourable because they are less expensive and can tolerate dark current contributions.

\footnotetext{
* Supported by GSI Darmstadt, EU and by BMBF, contr. no. 06F134I
}

To demonstrate the capabilites of the CH-DTL, it is foreseen to test an $\mathrm{sc} \mathrm{CH}$ cavity prototype. A design and engineering study has been performed in close cooperation with industry ${ }^{1}$. This study showed the feasibility of the production of superconducting $\mathrm{CH}$ cavities. The cavity production started in 2003; the completed cavity was delivered in April $2005[2]$

\begin{tabular}{|l|c|}
\hline frequency $[\mathrm{MHz}]$ & 352 \\
beta & 0.1 \\
$\mathrm{R}_{a} / \mathrm{Q}[\mathrm{k} \Omega]$ & 3.18 \\
$\mathrm{E}_{\text {peak }} / \mathrm{E}_{a}$ & $6.16 / 4.87$ \\
$\mathrm{~B}_{\text {peak }} / \mathrm{E}_{a}[\mathrm{mT} / \mathrm{MV} / \mathrm{m}]$ & $7.94 / 6.27$ \\
cavity length $[\mathrm{m}]$ & 1.048 \\
gaps & 19 \\
aperture diameter $[\mathrm{mm}]$ & 25 \\
tank diameter $[\mathrm{m}]$ & 0.28 \\
stem width a $[\mathrm{mm}]$ & 12.5 \\
stem width b [mm] & 20 \\
\hline
\end{tabular}

Table 1: Parameters of the prototype cavity (full/active length) ( $a$ and $b$ see fig. 6 )

\section{COUPLER DEVELOPMENT FOR THE PROTOTYPE}

For coupling into superconducting structures the external Q-value must cover the range from $10^{6}$ to $10^{9}$. Different kinds of couplers were examined with MicroWave Studio [3] and measured at a copper model[4].

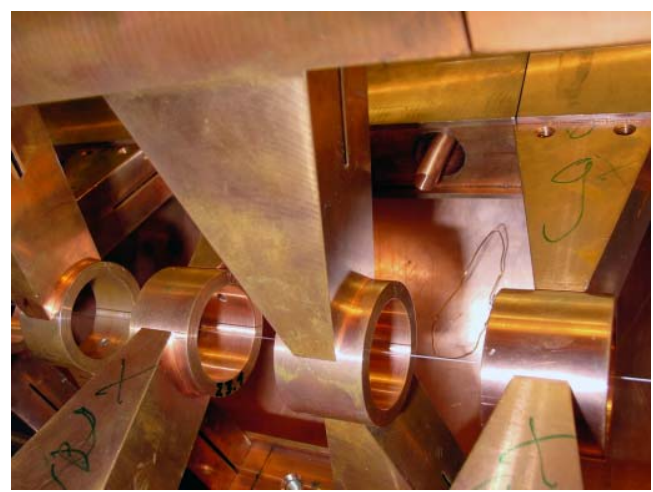

Figure 2: Capacitive coupling through one girder between two stems in the same plane

\footnotetext{
${ }^{1}$ ACCEL Company, Bergisch Gladbach, Germany
} 
Finally a capacitive coupling (fig.2) through the girder of the $\mathrm{CH}$-structure was chosen and studied in more detail. The inner conductor of the coaxial line is facing a drift tube with opposite polarity.

It turned out that by this method external Q-values from $10^{4}$ to $10^{11}$ can be achieved (fig.3). In order to verify the calculations with MicroWave Studio, measurements on the copper model were performed. These show a good agreement with the calculations.

After getting confidence in the simulations from the

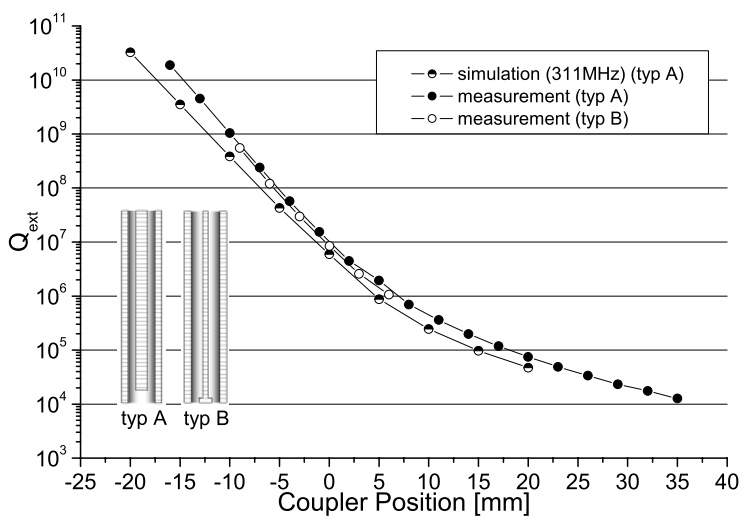

Figure 3: Calculations and measurements on capacitive couplers for the copper-model (Fig.2) of the CH-Cavity. $0 \mathrm{~mm}$ corresponds to the girder surface plane.

comparison with model measurements, calculations for the superconducting prototype of the $\mathrm{CH}$ structure were performed. The results of the calculations are represented in figure 4.

It showed that with a coupler position between -10 and $+10 \mathrm{~mm}$ external Q-values between $10^{5}$ and $10^{9}$ can be achieved (fig.4).

Figure 5 shows the coaxial coupler for the first cold tests of the sc CH-Cavity.

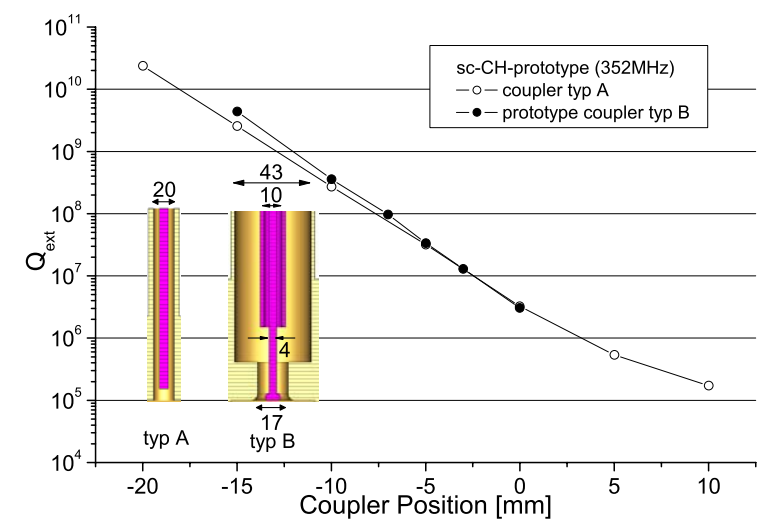

Figure 4: Calculations on capacitive couplers for the s.c. prototype of the $\mathrm{CH}$-Cavity.

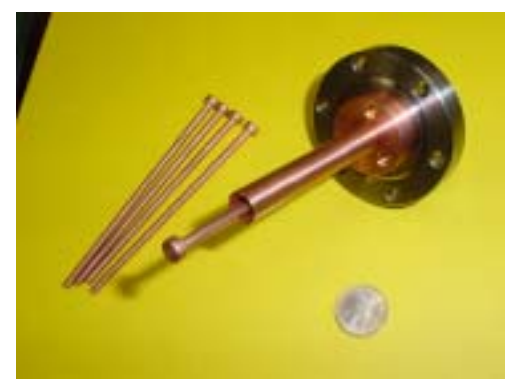

Figure 5: Coaxial coupler for the first cold tests.

\section{OPTIMIZATION FOR CAPACITIVE COUPLING}

In order to operate future $\mathrm{CH}$-cavities with more power, calculations were accomplished for the optimization of the girders and stems with MicroWave Studio.

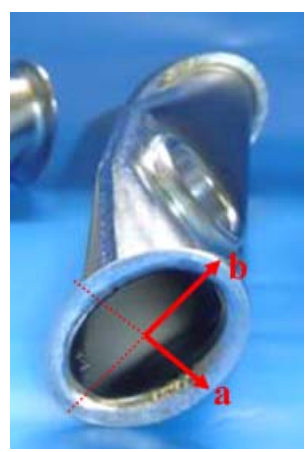

Figure 6: Stem of an sc CH-Cavity.

In the first step the girder geometry was changed to achieve a higher stability in relation to the vacuum forces. It became clear that the old stem form has some disadvantages for a girder variation, since it would lead to strong field increases (fig.7).

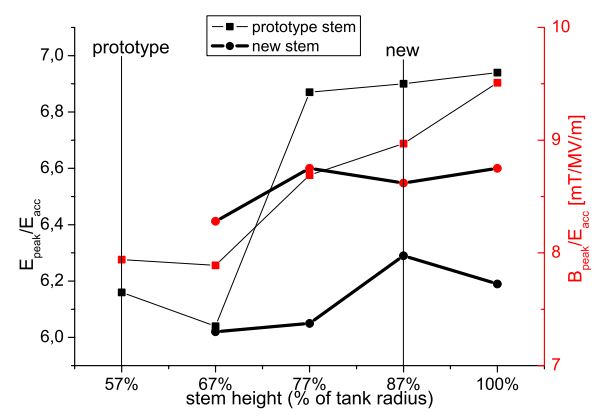

Figure 7: Electric and magnetic peak fields in dependence of the girder height

Geometry of the stems was changed to minimize the 
peak fields. In addition, the stem width in both directions $\mathrm{a}$ and $\mathrm{b}$ was varied. A widening of the stems led to $\mathrm{a}$ reduction of the fields (fig.8).

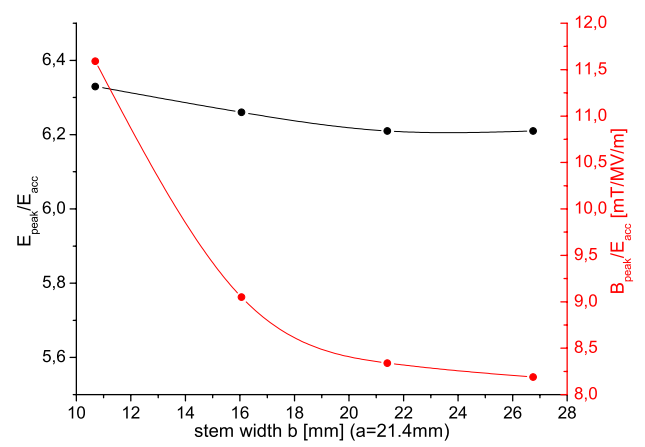

Figure 8: Electric and magnetic peak fields in dependence of the stem width a.

\section{CAPACITIVE COUPLING FOR THE OPTIMIZED SC CH-CAVITY}

After the optimization of the girder and stem geometry, we performed simulations for capacitive coupling. We use nearly the same position as for the prototype. Because of wider girders we can use a bigger coupler for high power. Figure 9 show the position of the new coaxial coupler. It can be seen that the coupler is not centered on the girder.

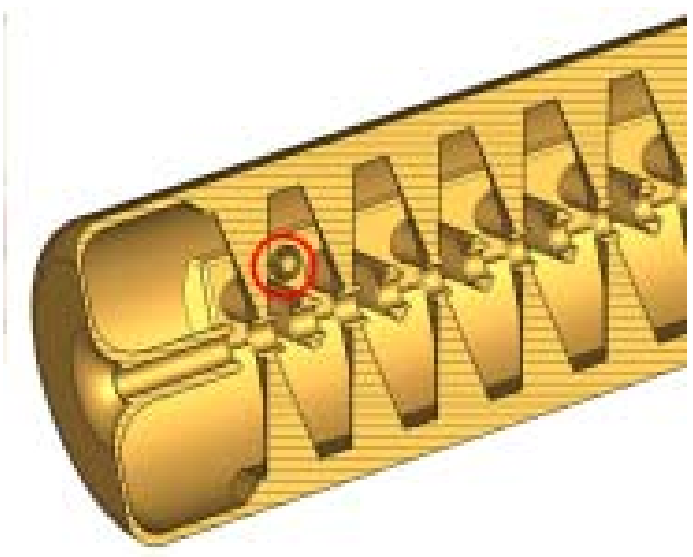

Figure 9: Optimized sc CH-Cavity for capacitive coupler

Figure 10 shows that with the new coupler it can be reach external Q-values between $10^{6}$ and $10^{8}$. So it is possible to use it for the sc CH-Cavity.

Table 2 shows that every parameter is nearly the same as for the prototype, but the new girder and stem geometry give the cavity more stiffness and there is more space for a larger coupler. With the new girder and stem it is possible to get more power into the $\mathrm{CH}$-cavity.

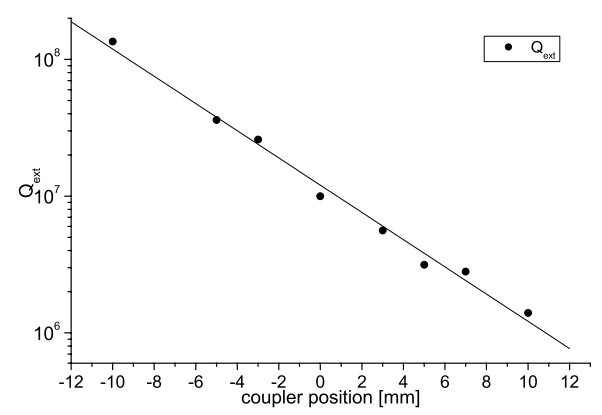

Figure 10: Qext for the optimized Cavity.

\begin{tabular}{|l|c|}
\hline frequency [MHz] & 352 \\
beta & 0.1 \\
$\mathrm{R}_{a} / \mathrm{Q}[\mathrm{k} \Omega]$ & 3.28 \\
$\mathrm{E}_{\text {peak }} / \mathrm{E}_{a}$ & $6.21 / 4.92$ \\
$\mathrm{~B}_{\text {peak }} / \mathrm{E}_{a}[\mathrm{mT} / \mathrm{MV} / \mathrm{m}]$ & $8.19 / 6.46$ \\
cavity length $[\mathrm{m}]$ & 1.048 \\
gaps & 19 \\
aperture diameter $[\mathrm{mm}]$ & 25 \\
tank diameter $[\mathrm{m}]$ & 0.28 \\
stem width a [mm] & 21.4 \\
stem width b [mm] & 27 \\
\hline
\end{tabular}

Table 2: Parameters of the optimized cavity for capacitive (full/active length).

\section{REFERENCES}

[1] H. Podlech, Development of Superconducting and Room Temperature CH-Structures, Proceeding of the LINAC 2004, Lübeck, Germany

[2] H. Podlech, First Tests of the Superconducting CH-Structure, these proceedings

[3] http://www.cst.com

[4] H. Liebermann, H. Podlech, U. Ratzinger, A. Sauer, Status of Coupler Development for the $352 \mathrm{MHz}$ Superconducting $\mathrm{CH}-$ Cavity, Proceeding of the LINAC 2004, Lübeck, Germany 